\title{
SYNTHESIS AND FREE RADICAL SCAVENGING PROPERTY OF SOME QUINOLINE DERIVATIVES
}

\author{
R. SUBASHINI, S. MOHANA ROOPAN, F. NAWAZ KHAN* \\ * Organic and Medicinal Chemistry Research Laboratory, Organic Chemistry Division, School of Advanced Sciences, \\ VIT University, Vellore 632 014, Tamil Nadu, India. \\ (Received: June 10, 2009 - Accepted: November 23, 2009)
}

\begin{abstract}
In the present work synthesis of 2-chloroquinoline-3-carbaldehydes (1a-g), 2-chloro-3-(1, 3-dioxolan-2-yl)quinolines (2a-g) and antioxidant activity using the DPPH assays is reported. The results showed that the compounds $\mathbf{1 b}, \mathbf{1 c}, \mathbf{2 b}, \mathbf{2 e}, \mathbf{2} \mathbf{f}$ possessed 84.65 to $85.75 \%$ radical scavenging activity where as compound 1g showing $92.96 \%$ radical scavenging activity.
\end{abstract}

Keywords: Quinoline derivatives, antioxidant, DPPH method

\section{INTRODUCTION}

Consumption of fruits and vegetables by human beings, shown lower risk of chronic diseases such as cancer, cardiovascular disease and stroke ${ }^{1}$ and positive health effects ${ }^{2}$ due to high contents of certain phenolic compounds in plant-derived foods. Recently, phytochemicals and their effects on human health have been intensively studied. In particular, a search for antioxidants ${ }^{3}$, hypoglycemic agents ${ }^{4}$, and anticancer agents ${ }^{5}$ in vegetables, fruits, tea, spices and medicinal herbs has attracted great attention. Naturally occurring plant phenolics include several groups of compounds that have health promoting properties. Phenolics may act as antioxidants, thereby reducing the risk of atherosclerosis and coronary heart disease, which can be caused by oxidation of low-density lipoproteins. They also may protect against some forms of cancer $^{6}$. Quinoline moiety is mostly present in a medicinal plant whose tubers possesses cardiovascular, anti-inflammatory and antiparasitic effects and has been used for the treatment of hepatitis, rheumatism and diarrhea? The importance of quinoline and its annulated derivatives is well recognized by synthetic and biological chemists. Quinoline is substantial molecular fragments of camptothecin are known to possess carcinogenic activity ${ }^{9}$.

In continuation of our work ${ }^{10}$, we undertook the comparative antioxidant activity of 2-chloroquinoline-3-carbaldehydes (1a-g) and 2-chloro-3-(1, 3-dioxolan-2-yl)quinolines (2a-g). (Fig 1).

\section{EXPERIMENTAL}

The materials were purchased from Sigma-Aldrich and Merck and were used without any additional purification. All reactions were monitored by thin layer chromatography (TLC). Melting points were determined in open capillaries using Dalal Melting Point Apparatus (Sunbim) and were corrected with respect to Benzoic acid. The FT-IR spectrum of samples was recorded on AVATAR 330 Thermo Nicolet spectrophotometer. ${ }^{1} \mathrm{H}$ NMR spectra were recorded using BRUKER $300 \mathrm{MHz}$ instrument and BRUKER NRC- IISc 400 $\mathrm{MHz}$ instrument (with TMS as internal reference). GCMS analyses were performed with Agilent GCMS- 5973 Inert MSD series.

General procedure for synthesis of 2-chloro-3-(1, 3-dioxolan-2-yl) quinolines (2a-g)

All the 2-chloroquinoline-3-carbaldehydes (1a-g) were prepared by the Meth-Cohn method ${ }^{11}$. A solution of 2-chloroquinoline-3-carbaldehydes 1 (10 $\mathrm{mmol})$ in benzene $(50 \mathrm{~mL})$ containing ethylene glycol $(1.78 \mathrm{~g}, 1.6 \mathrm{~mL}, 28.5$ $\mathrm{mmol}$ ) and a crystal of toluene- $p$-sulfonic acid was heated under reflux for 5 hours using a Dean-Stark water separator until no more water collected in the side arm. The cooled solution was treated with saturated aqueous sodium carbonate $(50 \mathrm{~mL})$, Benzene layer separated, dried and evaporated giving 2 which was recrystallised from petroleum ether gave a yellowish white solid. The product was characterized by NMR, GCMS techniques. Similar procedures were followed for the synthesis of other quinoline derivatives $\mathbf{2 a - g}$ and Table 1.

The spectral data of the compounds $\mathbf{1}(\mathbf{a}-\mathbf{g}) \& \mathbf{2}(\mathbf{a}-\mathbf{g})$ is given below.

2-Chloroquinoline-3-carbaldehyde ${ }^{12}$ (1a): Pale yellow powder, $82 \%$ yield, mp $148-150^{\circ} \mathrm{C}\left(\right.$ Lit. $\left.149^{\circ} \mathrm{C}\right)$. IR $(\mathrm{KBr}) \mathrm{cm}^{-1}: 3043,2923$ (Aromatic C-H), 2871(aldehyde $\mathrm{C}-\mathrm{H}), 1687(\mathrm{C}=\mathrm{O}), 1579(\mathrm{C}=\mathrm{N}), 761(\mathrm{C}-\mathrm{Cl}) .{ }^{1} \mathrm{H}$ NMR $\left(\mathrm{CDCl}_{3}\right.$ $400 \mathrm{MHz})$ d: 7.68-7.64 $(1 \mathrm{H}, \mathrm{m}, \mathrm{CH}), 7.92-7.87(1 \mathrm{H}, \mathrm{m}, \mathrm{CH}), 8.00-7.98(1 \mathrm{H}, \mathrm{d}$,
$\mathrm{J}=8 \mathrm{~Hz}, \mathrm{CH}), 8.09-8.07(1 \mathrm{H}, \mathrm{d}, J=8 \mathrm{~Hz}, \mathrm{CH}), 8.77(1 \mathrm{H}, \mathrm{s}, \mathrm{CH}), 10.57(1 \mathrm{H}, \mathrm{s}$, CHO). ESI-MS $m / z$ : 191( $\left.\mathrm{M}^{+}\right)$, Calcd for $\mathrm{C}_{10} \mathrm{H}_{6}$ CINO: 191.01 .

2-Chloro-8-methylquinoline-3-carbaldehyde ${ }^{12}$ (1b): Pale yellow powder, $77 \%$ yield, $\mathrm{mp} 136-138^{\circ} \mathrm{C}$ (Lit. $137^{\circ} \mathrm{C}$ ). IR $(\mathrm{KBr}) \mathrm{cm}^{-1}: 3050$, 2919(Aromatic C-H), 2869(aldehyde C-H), 1683(C=O), 1586(C=N), 772(CCl). ${ }^{1} \mathrm{H}$ NMR $\left(\mathrm{CDCl}_{3} 300 \mathrm{MHz}\right)$ d: $2.48\left(3 \mathrm{H}, \mathrm{s}, \mathrm{CH}_{3}\right), 7.64-7.60(2 \mathrm{H}, \mathrm{m}, \mathrm{CH})$, 7.89-7.85 (1H, m, CH $), 8.56(1 \mathrm{H}, \mathrm{s}, \mathrm{CH}), 10.45(1 \mathrm{H}, \mathrm{s}, \mathrm{CHO})$. ESI-MS $m / z$ : 205, Calcd for $\mathrm{C}_{11} \mathrm{H}_{8}$ ClNO: 205.64.

2-Chloro-7-methylquinoline-3-carbaldehyde ${ }^{12}$ (1c): Pale yellow powder, $86 \%$ yield, mp $144-146^{\circ} \mathrm{C}$ (Lit. $146^{\circ} \mathrm{C}$ ). IR $(\mathrm{KBr}) \mathrm{cm}^{-1}: 3045$, 2921(Aromatic C-H), 2868(aldehyde C-H), 1689(C=O), 1579(C=N), 811(C-Cl).

2-Chloro-6-methylquinoline-3-carbaldehyde ${ }^{12}$ (1d): Pale yellow powder, $80 \%$ yield, $\mathrm{mp} \quad 122-123^{\circ} \mathrm{C}$ (Lit. $123^{\circ} \mathrm{C}$ ). IR $\left(\mathrm{KBr} \mathrm{cm}^{-1}\right.$ : 3050 , 2921(Aromatic C-H), 2875(aldehyde C-H), 1691(C=O), 1579(C=N), 821(CCl). ${ }^{1} \mathrm{H}$ NMR $\left(\mathrm{CDCl}_{3} 400 \mathrm{MHz}\right) \mathrm{d}: 2.56(3 \mathrm{H}, \mathrm{s}, \mathrm{CH}), 7.26(1 \mathrm{H}, \mathrm{s}, \mathrm{CH}), 7.74-$ $7.70(1 \mathrm{H}, \mathrm{d}, J=10.6 \mathrm{~Hz}, \mathrm{CH}), 7.97-7.95(1 \mathrm{H}, \mathrm{d}, J=8.4 \mathrm{~Hz}, \mathrm{CH}), 8.66(1 \mathrm{H}, \mathrm{s}$, $\mathrm{CH}), 10.55(1 \mathrm{H}, \mathrm{s}, \mathrm{CHO})$. ESI-MS m/z: 205, Calcd for $\mathrm{C}_{11} \mathrm{H}_{8} \mathrm{ClNO}$ : 205.64.

2-Chloro-8-methoxyquinoline-3-carbaldehyde ${ }^{12}$ (1e): Pale yellow powder, $75 \%$ yield, $\mathrm{mp} 190-192^{\circ} \mathrm{C}\left(\mathrm{Lit} .190^{\circ} \mathrm{C}\right.$ ). IR $(\mathrm{KBr}) \mathrm{cm}^{-1}: 2940$ (Aromatic (C-H), 2866(aldehyde $\mathrm{C}-\mathrm{H}), 1688(\mathrm{C}=\mathrm{O}), 1584(\mathrm{C}=\mathrm{N}), 763(\mathrm{C}-\mathrm{Cl}) .{ }^{1} \mathrm{H}$ NMR $\left(\mathrm{CDCl}_{3} 400 \mathrm{MHz}\right) \mathrm{d}: 4.10\left(3 \mathrm{H}, \mathrm{s}, \mathrm{CH}_{3}\right), 7.27-7.22(1 \mathrm{H}, \mathrm{m}, \mathrm{CH}), 7.58-7.55(2 \mathrm{H}$, $\mathrm{m}, \mathrm{CH}), 8.72(1 \mathrm{H}, \mathrm{s}, \mathrm{CH}), 10.57(1 \mathrm{H}, \mathrm{s}, \mathrm{CHO})$. ESI-MS $m / z$ : 221, Calcd for $\mathrm{C}_{11} \mathrm{H}_{8} \mathrm{CINO}_{2}: 221.64$.

2-Chloro-6-methoxyquinoline-3-carbaldehyde ${ }^{12} \quad$ (1f): Pale yellow powder, $62 \%$ yield, mp $145-146^{\circ} \mathrm{C}$ (Lit. $\left.146^{\circ} \mathrm{C}\right)$. IR $(\mathrm{KBr}) \mathrm{cm}^{-1}: 3051$, 2922(Aromatic C-H), 1680(C=O), 1573(C=N), 814(C-Cl). ${ }^{1} \mathrm{H}$ NMR $\left(\mathrm{CDCl}_{3}\right.$ $300 \mathrm{MHz})$ d: $3.96\left(3 \mathrm{H}, \mathrm{s}, \mathrm{CH}_{3}\right), 7.20-7.19(1 \mathrm{H}, \mathrm{d}, \mathrm{CH}), 7.54-7.50(1 \mathrm{H}, \mathrm{m}, \mathrm{CH})$, 7.99-7.95 $(1 \mathrm{H}, \mathrm{d}, J=9.24 \mathrm{~Hz}, \mathrm{CH}), 8.65(1 \mathrm{H}, \mathrm{s}, \mathrm{CH}) 10.55(1 \mathrm{H}, \mathrm{s}, \mathrm{CHO})$. ESIMS $m / z: 221$, Calcd for $\mathrm{C}_{11} \mathrm{H}_{8} \mathrm{ClNO}_{2}: 221.64$

2-Chloro-7, 8-dimethylquinoline-3-carbaldehyde (1g): Pale yellow powder, $83 \%$ yield, $\mathrm{mp} 156{ }^{\circ} \mathrm{C}$. IR $(\mathrm{KBr}) \mathrm{cm}^{-1}$ : 2924(Aromatic C-H), 2864(aldehyde $\mathrm{C}-\mathrm{H}), 1686(\mathrm{C}=\mathrm{O}), 1590(\mathrm{C}=\mathrm{N}), 814(\mathrm{C}-\mathrm{Cl}) .{ }^{1} \mathrm{H}$ NMR $\left(\mathrm{CDCl}_{3}\right.$, $300 \mathrm{MHz}) \mathrm{d}: 2.54\left(3 \mathrm{H}, \mathrm{s}, \mathrm{CH}_{3}\right), 2.72\left(3 \mathrm{H}, \mathrm{s}, \mathrm{CH}_{3}\right), 7.46-7.44(1 \mathrm{H}, \mathrm{d}, J=8.25 \mathrm{~Hz}$, $\mathrm{CH}), 7.72-7.69(1 \mathrm{H}, \mathrm{d}, J=8.25 \mathrm{~Hz}, \mathrm{CH}), 8.65(1 \mathrm{H}, \mathrm{s}, \mathrm{CH}) 10.54(1 \mathrm{H}, \mathrm{s}, \mathrm{CHO})$. ESI-MS $m / z$ : 219, Calcd for $\mathrm{C}_{12} \mathrm{H}_{10}$ ClNO: 219.67 .

2-Chloro-3-(1,3-dioxolan-2-yl)quinoline ${ }^{13}$ (2a): Pale yellow powder, $65 \%$ yield, $\mathrm{mp} 60-62{ }^{\circ} \mathrm{C}$. IR $(\mathrm{KBr}) \mathrm{cm}^{-1}: 2899(\mathrm{C}-\mathrm{H}), 1621(\mathrm{C}=\mathrm{C}), 1567(\mathrm{C}=\mathrm{N}), 1330(-$ $\left.\mathrm{CH}_{2}\right), 1100(\mathrm{O}-\mathrm{C}), 755(\mathrm{C}-\mathrm{Cl}) .{ }^{1} \mathrm{H}$ NMR $\left(\mathrm{CDCl}_{3} 400 \mathrm{MHz}\right) \mathrm{d}: 4.20-4.13(4 \mathrm{H}, \mathrm{m}$, $\mathrm{CH}_{2} \mathrm{X}$ 2), $6.24(1 \mathrm{H}, \mathrm{s}, \mathrm{CH}), 7.59-7.55(1 \mathrm{H}, \mathrm{m}, \mathrm{CH}), 7.77-7.73(1 \mathrm{H}, \mathrm{m}, \mathrm{CH})$, 7.86-7.84 (1H, d, $J=8.4 \mathrm{~Hz}, \mathrm{CH}), 8.04-8.02(1 \mathrm{H}, \mathrm{d}, J=8.8 \mathrm{~Hz}, \mathrm{CH}), 8.40(1 \mathrm{H}, \mathrm{s}$, $\mathrm{CH})$. ESI-MS $m / z$ : 235, Calcd for $\mathrm{C}_{12} \mathrm{H}_{10} \mathrm{ClNO}_{2}: 235.67$.

2-Chloro-3-(1,3-dioxolan-2-yl)-8-methylquinoline ${ }^{13}(2 \mathrm{~b})$ : Pale yellow powder, $87 \%$ yield, $\mathrm{mp} 86-88^{\circ} \mathrm{C}$. IR $(\mathrm{KBr}) \mathrm{cm}^{-1}: 2919$ (Aromatic $\mathrm{C}-\mathrm{H}$ ), $2883(\mathrm{C}-\mathrm{H}), 1614(\mathrm{C}=\mathrm{C}), 1597(\mathrm{C}=\mathrm{N}), 1330(-\mathrm{CH}), 1101(\mathrm{O}-\mathrm{C}), 765(\mathrm{C}-\mathrm{Cl}) .{ }^{1} \mathrm{H}$ NMR $\left(\mathrm{CDCl}_{3} 400 \mathrm{MHz}\right)$ d: $2.78\left(3 \mathrm{H}, \mathrm{s}, \mathrm{CH}_{3}\right), 4.22-4.12\left(4 \mathrm{H}, \mathrm{m}, \mathrm{CH}_{2} \mathrm{X} 2\right), 6.26$ $(1 \mathrm{H}, \mathrm{s}, \mathrm{CH}), 7.49-7.44(1 \mathrm{H}, \mathrm{m}, \mathrm{CH}), 7.61-7.58(1 \mathrm{H}, \mathrm{d}, J=7 \mathrm{~Hz}, \mathrm{CH}), 7.70-7.68$ $(1 \mathrm{H}, \mathrm{d}, J=8.08 \mathrm{~Hz}, \mathrm{CH}), 8.37(1 \mathrm{H}, \mathrm{s}, \mathrm{CH})$. LC-MS $m / z: 250\left(\mathrm{M}^{+}\right)$, Calcd for $\mathrm{C}_{13} \mathrm{H}_{12} \mathrm{ClNO}_{2}: 249.69$.

2-Chloro-3-(1, 3-dioxolan-2-yl)-7-methylquinoline ${ }^{11}$ (2c): Pale yellow powder, $85 \%$ yield, $\mathrm{mp} 72-74^{\circ} \mathrm{C}\left(\mathrm{Lit} .75-76^{\circ} \mathrm{C}\right.$ ). IR $(\mathrm{KBr}) \mathrm{cm}^{-1}: 2921$ (Aromatic $\mathrm{C}-\mathrm{H}), 1626(\mathrm{C}=\mathrm{C}), 1541(\mathrm{C}=\mathrm{N}), 1330\left(-\mathrm{CH}_{2}\right), 1101(\mathrm{O}-\mathrm{C}), 810(\mathrm{C}-\mathrm{Cl}) .{ }^{1} \mathrm{H}$ NMR $\left(\mathrm{CDCl}_{3} 400 \mathrm{MHz}\right) \mathrm{d}: 2.58\left(3 \mathrm{H}, \mathrm{s}, \mathrm{CH}_{3}\right), 4.13-4.23\left(4 \mathrm{H}, \mathrm{m}, \mathrm{CH}_{2} \mathrm{X} 2\right), 6.24(1 \mathrm{H}$, 
s, CH), 7.44-7.42 (1H, d,J=8.4Hz, CH), 7.77-7.75 (1H, d, J=8 Hz, CH), 7.83 $(1 \mathrm{H}, \mathrm{s}, \mathrm{CH}), 8.37(1 \mathrm{H}, \mathrm{s}, \mathrm{CH})$. ESI-MS $m / z: 250[\mathrm{M}+1]$, Calcd for $\mathrm{C}_{13} \mathrm{H}_{12} \mathrm{ClNO}_{2}$ : 249.69 .

2-Chloro-3-(1,3-dioxolan-2-yl)-6-methylquinoline ${ }^{13}$ (2d): Pale yellow powder, $78 \%$ yield, mp $52-54^{\circ} \mathrm{C}$. IR $(\mathrm{KBr}) \mathrm{cm}^{-1}: 2894(\mathrm{C}-\mathrm{H}), 1598(\mathrm{C}=\mathrm{N})$, 1329(-CH $), 1096(\mathrm{O}-\mathrm{C}), 823(\mathrm{C}-\mathrm{Cl}) .{ }^{1} \mathrm{H}$ NMR $\left(\mathrm{CDCl}_{3} 300 \mathrm{MHz}\right) \mathrm{d}: 2.54(3 \mathrm{H}$, s, $\left.\mathrm{CH}_{3}\right), 4.20-4.13\left(4 \mathrm{H}, \mathrm{m}, \mathrm{CH}_{2} \mathrm{X} 2\right), 6.23(1 \mathrm{H}, \mathrm{s}, \mathrm{CH}), 7.62-7.57(2 \mathrm{H}, \mathrm{m}, \mathrm{CH})$, 7.95-7.92 (1H, d, $J=8.55 \mathrm{~Hz}, \mathrm{CH}), 8.32(1 \mathrm{H}, \mathrm{s}, \mathrm{CH})$. ESI-MS $m / z: 250[\mathrm{M}+1]$, Calcd for $\mathrm{C}_{13} \mathrm{H}_{12} \mathrm{ClNO}_{2}: 249.69$

2-Chloro-3-(1,3-dioxolan-2-yl)-8-methoxyquinoline ${ }^{13}$ (2e): Pale yellow powder, $82 \%$ yield, mp $119-121^{\circ} \mathrm{C} .{ }^{1} \mathrm{H}$ NMR $\left(\mathrm{CDCl}_{3} 300 \mathrm{MHz}\right)$ d: $4.10(3 \mathrm{H}, \mathrm{s}$, $\left.\mathrm{OCH}_{3}\right), 4.32-4.20\left(4 \mathrm{H}, \mathrm{m}, \mathrm{CH}_{2} \mathrm{X} 2\right), 6.32(1 \mathrm{H}, \mathrm{s}, \mathrm{CH}), 7.27-7.22(1 \mathrm{H}, \mathrm{m}, \mathrm{CH})$, 7.58-7.55 (2H, m, CH), $8.72(1 \mathrm{H}, \mathrm{s}, \mathrm{CH})$. IR $(\mathrm{KBr}) \mathrm{cm}^{-1}: 1632,1107,805$. ESIMS $m / z: 266[\mathrm{M}+1]$, Calcd for $\mathrm{C}_{13} \mathrm{H}_{12} \mathrm{ClNO}_{3}: 265.69$.

2-Chloro-3-(1,3-dioxolan-2-yl)-6-methoxyquinoline (2f): Pale yellow powder, 74\% yield, mp 93-95 ${ }^{\circ} \mathrm{C}$. IR $(\mathrm{KBr}) \mathrm{cm}^{-1}$ : 2930(Aromatic $\mathrm{C}-\mathrm{H}$ ), $1619(\mathrm{C}=\mathrm{C}), 1594(\mathrm{C}=\mathrm{N}), 1332\left(-\mathrm{CH}_{2}\right), 1103(\mathrm{O}-\mathrm{C}), 820(\mathrm{C}-\mathrm{Cl}) .{ }^{1} \mathrm{H} \mathrm{NMR}\left(\mathrm{CDCl}_{3}\right.$ $300 \mathrm{MHz})$ d: $3.92\left(3 \mathrm{H}, \mathrm{s}, \mathrm{OCH}_{3}\right), 4.22-4.10\left(4 \mathrm{H}, \mathrm{m}, \mathrm{CH}_{2} \mathrm{X} 2\right), 6.22(1 \mathrm{H}, \mathrm{s}$, $\mathrm{CH}), 7.11-7.10(1 \mathrm{H}, \mathrm{d}, J=2.43 \mathrm{~Hz}, \mathrm{CH}), 7.41-7.37(1 \mathrm{H}, \mathrm{m}, \mathrm{CH}), 7.94-7.91$ $(1 \mathrm{H}, \mathrm{d}, J=9.18 \mathrm{~Hz}, \mathrm{CH}), 8.30(1 \mathrm{H}, \mathrm{s}, \mathrm{CH})$. ESI-MS $m / z: 266[\mathrm{M}+1]$, Calcd for $\mathrm{C}_{13} \mathrm{H}_{12} \mathrm{ClNO}_{3}: 265.69$.

2-Chloro-3-(1,3-dioxolan-2-yl)-7,8-dimethylquinoline

$(2 \mathrm{~g})$ Pale yellow powder, $81 \%$ yield, mp $107-109{ }^{\circ} \mathrm{C}$. IR $(\mathrm{KBr}) \mathrm{cm}^{-1}: 3053$, $2887(\mathrm{C}-\mathrm{H}), 1606(\mathrm{C}=\mathrm{C}), 1563(\mathrm{C}=\mathrm{N}), 1360\left(-\mathrm{CH}_{2}\right), 1108(\mathrm{O}-\mathrm{C}), 781(\mathrm{C}-\mathrm{Cl}),{ }^{1} \mathrm{H}$ $\operatorname{NMR}\left(\mathrm{CDCl}_{3} 300 \mathrm{MHz}\right)$ d: $2.54\left(3 \mathrm{H}, \mathrm{s}, \mathrm{CH}_{3}\right), 2.72\left(3 \mathrm{H}, \mathrm{s}, \mathrm{CH}_{3}\right), 4.22-4.10(4 \mathrm{H}$, $\left.\mathrm{m}, \mathrm{CH}_{2} \mathrm{X} 2\right)^{2}, 6.22(1 \mathrm{H}, \mathrm{s}, \mathrm{CH}), 7.46-7.44(1 \mathrm{H}, \mathrm{d}, J=8.25 \mathrm{~Hz}, \mathrm{CH}), 7.72-7.69$ $(1 \mathrm{H}, \mathrm{d}, J=8.25 \mathrm{~Hz}, \mathrm{CH}), 8.65(1 \mathrm{H}, \mathrm{s}, \mathrm{CH})$. ESI-MS $m / z: 264[\mathrm{M}+1]$, Calcd for $\mathrm{C}_{14} \mathrm{H}_{14} \mathrm{ClNO}_{2}: 263.72$.

\section{BIOLOGICAL INVESTIGATION}

Preparation of stock solution

The synthesized compounds were used to prepare stock using ethanol $(0.3$ $\mathrm{mM})$. The appropriate concentrations of the compounds were made by serial dilution.

\section{Invitro antioxidant activity}

Antioxidant activity was measured by following methodology assessed by Roopan et al., ${ }^{14,15}$. For the present study the samples were prepared in different concentrations i.e. $0.02,0.05,0.1,0.15,0.2 \mathrm{mM}$ in AR grade ethanol. The samples $(3 \mathrm{~mL})$ of above concentrations were mixed with $1 \mathrm{~mL}$ of $0.15 \mathrm{mM}$ of DPPH prepared in AR grade ethanol and incubated at room temperature for 30 min in dark. The absorbance of the incubated solutions and the blank (with out sample) were recorded against BHT. The absorbance was measured at $517 \mathrm{~nm}$ using a UV-Visible Spectrophotometer. Radical Scavenging Capacity (RSC) in percent was calculated by the following equation:

$\begin{array}{lll}\mathbf{R S C}(\mathbf{\%}) & = & \mathbf{1 0 0} \times\left(\mathbf{A}_{\text {blank }}-\mathbf{A}_{\text {sample) } /} \mathbf{A}_{\text {blank }}\right. \\ \text { Where } & & \\ \text { RSC } & = & \text { Radical Scavenging Capacity } \\ \mathrm{A}_{\text {blank }} & = & \text { Absorbance of blank } \\ \mathrm{A}_{\text {blank }} & = & \text { Absorbance of sample }\end{array}$

\section{RESULTS AND DISCUSSION}

Radical scavenging activity is very important due to the deleterious role of free radicals in foods and in biological systems. Diverse methods are currently used to assess the antioxidant activity. Herein is reported a synthesis of 2-chloro-quinoline-3-carboxaldehydes (1a-g) and 2-chloro-3-(1, 3 -dioxolan-2-yl)quinolines (2a-g) as potential antioxidants. Chemical assays are based on the ability to scavenge synthetic free radicals, using a variety of radical-generating systems and methods for detection of the oxidation endpoint. $\mathrm{DPPH}^{\times}$radical scavenging methods are common spectrophotometric procedures for determining the antioxidant capacities of components. All the compounds were fully characterized and screened for biological activity using DPPH method as per the literature methodology $y^{14,15}$. These assays have been applied to determine the antioxidant activity of pure component.
Table 1. Synthesis of 2-chloro-3-(1, 3-dioxolan-2-yl)quinoline, 2(a-g)<smiles>[R]c1c(C2OCCO2)cc2cc(C=O)c(Cl)nc2c1[R]</smiles>

\begin{tabular}{cccc} 
1 and 2 & $\mathrm{R}$ & $\mathrm{R}^{\prime}$ & $\mathrm{R}{ }^{\prime \prime}$ \\
\hline $\mathrm{a}$ & $\mathrm{H}$ & $\mathrm{H}$ & $\mathrm{H}$ \\
$\mathrm{b}$ & $\mathrm{CH}_{3}$ & $\mathrm{H}$ & $\mathrm{H}$ \\
$\mathrm{c}$ & $\mathrm{H}$ & $\mathrm{CH}_{3}$ & $\mathrm{H}$ \\
$\mathrm{e}$ & $\mathrm{H}$ & $\mathrm{H}$ & $\mathrm{CH}_{3}$ \\
$\mathrm{f}$ & $\mathrm{OCH}_{3}$ & $\mathrm{H}$ & $\mathrm{H}$ \\
$\mathrm{g}$ & $\mathrm{H}$ & $\mathrm{H}$ & $\mathrm{OCH}_{3}$ \\
& $\mathrm{CH}_{3}$ & $\mathrm{CH}_{3}$ & $\mathrm{H}$ \\
\hline
\end{tabular}

When an antioxidant added to the radicals there is a degree of decolorization owing to the presence of the antioxidants which reverses the formation of the $\mathrm{DPPH}^{\times}$radical. Bleaching rate of a stable free radical, $\mathrm{DPPH}^{\times}$is monitored at a characteristic wavelength in the presence of the sample. In its radical form, $\mathrm{DPPH}^{\times}$absorbs at $517 \mathrm{~nm}$, but upon reduction by an antioxidant species its absorption decreases. When the absorption decreases the \% inhibition value will be increase. The absorbance was measured at $517 \mathrm{~nm}$. Different derivatives of quinoline reduced DPPH radicals significantly. Activities of quinoline (1a-g/2a-g) derivatives were compared with commercial antioxidant butylated hydroxy toluene, (BHT) (Fig. 1). DPPH is a stable free radical and accepts an electron or hydrogen radical to become a stable diamagnetic molecule. In the radical form, this molecule had an absorbance at $517 \mathrm{~nm}$ which disappeared after acceptance of an electron or hydrogen radical from an antioxidant compound to become a stable diamagnetic molecule. Compound $\mathbf{1 g}$ had relatively high DPPH radical scavenging activity at $0.2 \mathrm{mM}$ concentration. As shown in Fig. 1, 1b, 1d, $\mathbf{1 g} \& \mathbf{2 b}, \mathbf{2 e}, \mathbf{2} \mathbf{f}$, found to have the ability to scavenge hydroxyl radicals, remaining compounds are inactive.

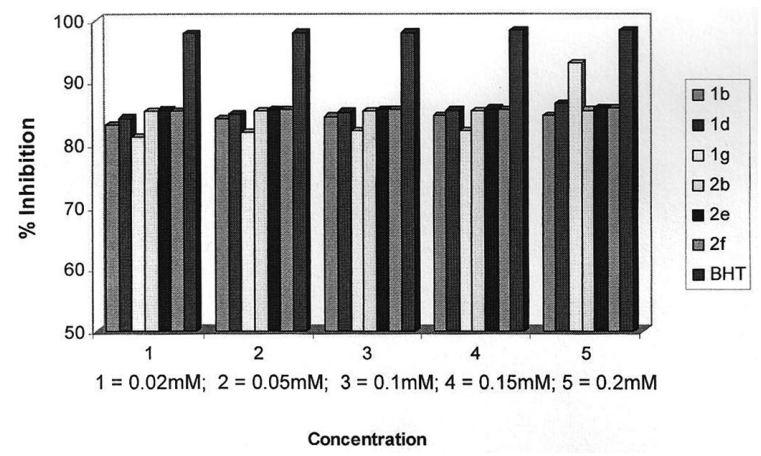

Fig 1. In vitro antioxidant activity of quinoline compounds 


\section{CONCLUSION}

In conclusion, we have developed a new anti-oxidative quinoline derivatives, 1a-g/2a-g. The maximum antioxidant activity was observed with 1g. Compounds 2e, $2 \mathrm{f}$ showed good free radical scavenging property and compounds $\mathbf{1 b}, \mathbf{1 d}$ exhibited less activity compare to other compounds.

\section{ACKNOWLEDGEMENT}

We are grateful for financial support from the Department of Science and Technology, Government of India (Grant No. SR/FTP/CS-99/2006) and VIT University, Vellore for providing research facilities. The authors wish to express their gratitude to Technology Buisness Incubator, VIT University, Madras Univeristy, Chennai, Indian Institute of Science, Bangalore and Syngene International Limited, Bangalore for their support of FT-IR, NMR, LCMS.

\section{REFERENCES}

1. K.J. Yeum, G. Aldini, H.Y. Chung, N.I. Krinsky, R.M. Russell, J. Nutr. 133, 2688, (2003).

2. Y.L. You, X.W. Duan, X.Y. Wei, X.G. Su, M.M. Zhao, J. Sun, N. Ruenroengklin, Y.M. Jiang, Molecules 12, 842, (2007).
3. G. Madhumitha, A.M. Saral, Asian J. Chem. 3, 2468, (2009).

4. H. Lü, J. Chen, W.L. Li, B.R. Ren, J.L. Wu, H.Y. Kang, H.Q. Zhang, A. Adams, N.D. Kimpe, J. Ethanopharmacol. 122, 486, (2009).

5. P.K. Raveedran Nair, S.J. Melnick, S.F. Wnuk, M. Rapp, E. Escalon, C. Ramachandran, J. Ethanopharmacol. 122, 450, (2009).

6. C.L. Emmons, D.M. Peterson, Crop Sci. 41, 1676, (2001)

7. G. Bourdy, C. Quenevo, A. Gimenez, Tacana, conozcan nuestros arboles, nuestras hierbas, Plural, La Paz : 414-416, (1999).

8. S.J. Teague, A.M. Davis, P.D. Leeson, T. Oprea, Angew. Chem. Int. Edit. 38, 3743, (1999).

9. R.W. Armstrong, A.P. Combs, P.A. Tempest, S.D. Brown, T.A. Keating, Accounts Chem. Res. 29, 123, (1996).

10. R.K. Somvanshi, R.Subashini, V.Dhanasekaran, G.Arulprakash, S.N.Das, S.Dey, J Chem Crystallogr.. 38, 381, (2008); S.M. Roopan, F.N. Khan, B.K. Mandal, Teltrahedron Lett., 51, 2309, (2010); S.M. Roopan, F.N. Khan, ARKIVOC, xiii, 161 (2009)

11. O. Meth-Cohn, B. Narine, B. Tarnowski, J. Chem. Soc. Perkin (I). 1520, (1981).

12. A. Srivastava, R.M. Singh, Indian J Chem. 44B, 1868, (2005).

13. Arash Afghan, M. Mehdi Baradarani, John A. Joule, Arkivoc. (ii), 20, (2009).

14. S.M. Roopan, T. Maiyalagan, F.N. Khan, Can J Chem. 86, 1019, (2008).

15. S.M. Roopan, F.N. Khan, Indian J. Heterocy. Ch. 18, 183, (2008). 